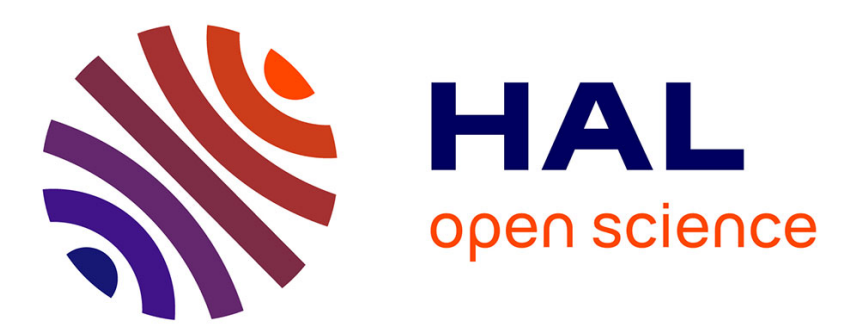

\title{
L'ancrage de l'évaluation des politiques publiques dans les sciences sociales aux États-Unis : reconstruction d'un linéament intellectuel
}

\author{
Vincent Claude Spenlehauer
}

\section{- To cite this version:}

Vincent Claude Spenlehauer. L'ancrage de l'évaluation des politiques publiques dans les sciences sociales aux États-Unis : reconstruction d'un linéament intellectuel. Revue française d'administration publique, 2013, 4, pp.324. 10.3917/rfap.148.0877 . hal-01903601

\section{HAL Id: hal-01903601 \\ https: / hal-enpc.archives-ouvertes.fr/hal-01903601}

Submitted on 24 Oct 2018

HAL is a multi-disciplinary open access archive for the deposit and dissemination of scientific research documents, whether they are published or not. The documents may come from teaching and research institutions in France or abroad, or from public or private research centers.
L'archive ouverte pluridisciplinaire HAL, est destinée au dépôt et à la diffusion de documents scientifiques de niveau recherche, publiés ou non, émanant des établissements d'enseignement et de recherche français ou étrangers, des laboratoires publics ou privés. 


\title{
L'ancrage de l'évaluation des politiques publiques dans les sciences sociales aux Etats-Unis : une proposition de linéament intellectuel
}

\author{
Vincent Spenlehauer
}

\section{Résumé}

Dans cet article, nous défendons l'idée que l'embarras des sciences sociales américaines eu égard à la notion d'Etat et à l'éventualité d'une étatisation de la société américaine a suscité l'invention d'une théorie de l'Etat qui ne dit pas son nom, l'analyse des politiques publiques, dont l'évaluation des politiques publiques est des avatars.

\section{Mot clés}

Sciences sociales américaines, science politique, théorie de l'Etat pragmatique, analyse et évaluation des politiques publiques, Woodrow Wilson, Walter Lippmann, John Dewey, Charles Merriam, Harold Lasswell, Aaron Wildavsky.

\section{Introduction : le paradigme américain de la public policy comme matrice de la policy evaluation}

En vue d'une éventuelle sociohistoire du concept d'évaluation de politique [Payre, Pollet 2013], nous tentons ici de mettre en évidence un linéament intellectuel et politique liant les sciences sociales américaines et plus spécialement l'œuvre de certains politistes américains (Wilson, Merriam, Lasswell et Wildavsky) avec la naissance de la policy evaluation. ${ }^{1}$, Il semble en effet que l'institutionnalisation et la professionnalisation de l'activité de policy evaluation aux États-Unis à la fin des années 1960 n’ont pu advenir qu'en

\footnotetext{
${ }^{1}$ Cet essai valorise un matériau oral et écrit récolté lors de séjours.aux Etats-Unis, notamment dans le cadre de bourses Fulbright et CNRS aux Universités de Californie à Los Angeles et Santa Barbara en 2000.
} 
raison de la construction à partir de la fin du $19^{\text {ème }}$ siècle par les sciences politiques états-uniennes de la catégorie de pensée social-scientifique «public policy»? Existe en arrière-fond de ce processus un rapport complexe et hypocrite des politistes américains à l'idée d'État qui, pour le coup, n’est pas mythifiée comme en Europe (Cassirer 1944)². On peut considérer que la pratique U.S. de la policy analysis \& evaluation incarne une tacite théorie américaine (en faveur) de l'État pragmatique. ${ }^{3}$ En fait, la force de l'État fédéral et le degré de souveraineté octroyée aux États fédérés sans menacer l'unité nationale a toujours fait l'objet de discussions et même d'une guerre meurtrière, celle de sécession (1861-65).

Pour l'historien et politiste James Farr (1993), cette discussion est au fondement de la science politique américaine en tant que discipline académique, via notamment le livre «On Civil Liberty and Self-Government» (1853) de Francis Lieber, un immigré prussien spécialiste de "statistik» hégélienne et premier professeur de science politique états-unien (à Columbia). Nous transposons cette hypothèse au cas particulier de la construction de la catégorie de pensée social-scientifique et d'action politique "public policy», sur un mode narratif centré sur l'intervention de quelques figures-clés de la science politique américaine. Nous évoquons tout d'abord Woodrow Wilson qui fait de la mise en place d'administrations publiques apolitiques et professionnelles la mission cardinale de la science politique. Vient ensuite, dans l'entre-deux-guerres, Charles Merriam qui

\footnotetext{
${ }^{2}$ On peut inversement penser avec C. W. Mills (1956) que des auteurs comme Robert Dahl mythifient le pluralisme du système politique américain. Voir aussi l'article de W.J. Novak (2008) sur le mythe du faible État américain, article qui sous-estime cependant la force de structuration politique de ce mythe. La California Highway Patrol fournit un exemple éloquent. De par sa taille, ses moyens et son action, elle joue dans les gouvernances policières californienne et américaine un rôle important, à la mesure des enjeux culturels, sociaux, politiques et économiques de cet État clé qu'est la Californie ; et bat en brèche l’idée qu'en raison d'un attachement viscéral au principe de self-government la chose policière aux États-Unis relève essentiellement de dynamiques locales (e.g. NYPD, LAPD...)[Hamelin, Spenlehauer 2006]. Pourtant, bien que généraliste et faisant donc bien davantage que de sécuriser les routes de Californie, la CHP continue à s'appeler modestement CHP. L'État américain, États fédérés inclus, s'est renforcé tout au long du 20 ème siècle, mais le localisme politique garde de très beaux restes du fait d'une culture très vivante de son mythe.

${ }^{3}$ La précocité de l'éclatement des sciences sociales américaines en plusieurs disciplines professionnalisées, seconde moitié du 19'me siècle (Haskell 2000), n’a pas empêché la science politique américaine de se positionner durablement comme une discipline carrefour ouverte aux autres sciences humaines (histoire, psychologie, philosophie...) et sociales. Si la science politique américaine constitue la matrice institutionnelle de la policy orientation le développement de cette dernière est scientifiquement redevable de divers développements social-scientifiques américains comme ceux, majeurs, de la sociologie des organisations ou de l'économie institutionnelle.
} 
positionne cette même science politique en tant que pôle de valorisation gouvernementale des autres disciplines des sciences humaines et sociales en vue de la mise en place d'un Etat tourné vers le public-problem-solving. Après 1945, son élève Harold Lasswell se démarque du pragmatisme étatique de son maitre pour envisager l'avènement de policy sciences démocratiques, lesquelles sont plus ou moins fidèlement institutionnalisées au début des années 1970 à travers l'ouverture de public policy schools \& masters programs dans de nombreuses universités américaines, Aaron Wildavsky étant le parangon de cette dernière étape entourant l'explosion de l'industrie évaluative aux Etats-Unis.

Rendre sans ambages l'épaisseur historique de l'évaluation aux Etats-Unis, ainsi que le rôle de matrice qu'ont joué et continuent à jouer les sciences sociales peut bien entendu alimenter le débat sur le retard français et sa « rattrapabilité ». Les pages qui suivent ne traitent que des Etats-Unis, mais, au sujet d'une prétendue avance américaine en matière évaluative, nous attirons l'attention du lecteur sur deux faits caractéristiques de la situation américaine. Primo, la problématique du pouvoir excessif du "Big Business», centrale pour Wilson, est désormais marginale pour les théoriciens et praticiens américains de l'évaluation. Du coup, la question de la reproduction par l'évaluation du déséquilibre des pouvoirs dans la fabrique de l'action publique n'est jamais posée. Secundo, le juste impératif d'interdisciplinarité dans la pratique de l'évaluation, et plus largement dans celle de la policy analysis, n’a jamais réellement dépassé le stade du vœu pieux.

\section{Wilson: point de départ: l'Etat organique sauveur du self- government}

Selon James Farr (1993), après la guerre de sécession, les fédéralistes l'ayant gagnée, l'Amérique s'urbanisant et s'industrialisant, le statisme américain se revigore en s'adjoignant une dimension bureaucratique. Woodrow Wilson (1856-1924), professeur de science politique, est la grande figure de ce néostatisme (Hofstadter 1955). Bien que démocrate 4 et donc attaché au principe de self-government du $10^{\text {ème }}$ amendement de la constitution, Wilson soutient

\footnotetext{
4 Sous cette étiquette, il sera entre autre élu gouverneur du New Jersey en 1910, puis Président des ÉtatsUnis en 1912.
} 
que les citoyens américains ne sont plus en mesure de contrôler leurs élus et les relations de ceux-ci avec le «big business». Par ailleurs, ces élus peinent à gouverner sans aide professionnelle tant la technicité de certains dossiers s'est accrue. A tout niveau, des administrations publiques composées d'agents compétents car bien formés et soustraits à l'habituel système des dépouilles (spoil system) devraient, selon Wilson, se mettre en place (Skowronek 1982). Darwiniste institutionnel, Wilson voit dans l'État organique une évolution salutaire du système politique américain: "The science of administration is the latest fruit of that study of politics which has begun some twenty-two bundred years ago. [...] Why did it wait till this too busy century of ours to demand attention to itself? Administration is the most obvious part of government; it is government in action [...]. The question was always: Who shall make law, and what shall that law be? The other question: how law should be administered with enlightenment, with equity, with speed, and without friction, was put aside as "practical detail" which clerks could arrange after doctors had agreed upon principles." (Wilson 1887).

Avant le tournant bureaucratique de Wilson, la science politique ne valait professionnellement que par le truchement de l'éducation civique. Avec les administrative studies, la science politique américaine se fixe non seulement un objet de recherche nouveau, mais également un objet d'expertise et d'intervention dans les affaires gouvernementales locales ou nationales (Ricci 1984). Une consécration de cette envolée académique et professionnelle a lieu avec la création en 1916, sous l'égide du président Wilson et de deux collègues politistes, Goodnow et Willoughby, de l'Institute for Government Research (devenu depuis un très important think tank: la Brookings Institution $)^{5}$.

C'est paradoxalement en minimisant la dimension politique de l'administration publique et en en magnifiant la dimension technique que la science politique américaine devient une discipline de sciences sociales établie et disposant d'un accès facile au cœur de l'activité gouvernementale, de la petite municipalité du Midwest à la Maison Blanche.

\footnotetext{
${ }^{5}$ On compte alors, dans les années 1910, une douzaine de départements universitaires de science politique (120 en 2007) produisant une vingtaine de $\mathrm{PhD}$ par an (1300 d'aujourd'hui), les deux premiers étant Columbia (NY) et l'Université de Chicago (Masuaka et al. 2007).
} 


\section{De l'État bureaucratiquement "bon d'office " à l'État résolvant des problèmes sociaux sur lesquels il faut enquêter}

L'après-1918 voit s'ouvrir une nouvelle ère pour les politistes états-uniens. Sans renier l'orientation bureaucratique des Wilson, Goodnow ou Willoughby (nés vers 1860), leurs successeurs ne peuvent ignorer que certaines disciplines voisines, la sociologie, l'économie, la psychologie ou la statistique développent des méthodologies d'observation pouvant transformer la science politique en science moderne (Gunnel 1992). Cette soif de ressourcement scientifique est renforcée par l'ébranlement de la foi de nombreux politistes américains en la solidité des institutions démocratiques de leur pays.

Walter Lippmann, à travers deux essais, Public Opinion (1922) et The Phantom Public (1924 jette le plus gros pavé dans la mare des fidèles. Observant combien les opinions publiques ont été, en Europe et aux États-Unis, pendant la guerre, facilement manipulées par les gouvernements et les médias il remet en cause le principe fondamental de la souveraineté populaire, le sacré «We the People». Pour lui, la fonction démocratique des citoyens n'intervient que pour sanctionner a posteriori, par le vote et avec plus ou moins de bonheur, les actions que l'élite au pouvoir a déjà conçues et entreprises sans leur concours. Lippmann ne réduit pas la citoyenneté démocratique à néant, mais infère que le caractère démocratique du système politique américain ne garantit pas seul la bonne édification du "public interest $»$. Dans son essai de 1922, Lippmann plaide pour l'émergence chez les insiders de l'action gouvernementale - fonctionnaires, chercheurs en sciences sociales, journalistes spécialisés,... - d'une "organized intelligence» permettant aux gouvernants de mieux faire le bonheur des gens sans leur avis préalable.

A la théorie minimaliste et élitiste de la démocratie de Lippmann on oppose souvent celle du philosophe pragmatiste John Dewey (1859-1952) dans son livre «The Public and Its Problems» (1927) (abrégé PIP ci-après). Dans « How We think» (1910), Dewey affirme que tout citoyen dispose d'une capacité «d'intellectualisation» des problèmes auquel il est confrontée. En

\footnotetext{
${ }^{6} \mathrm{Il}$ propose un schéma en " five logically distinct steps : (i) a felt difficulty; (it) its location and definition; (tit) suggestion of possible solution; (tv) development by reasoning of the bearings of the suggestions; (v) further observation and experiment leading to its acceptance or rejection; that is, the conclusion of belief or disbelief' (p.72). La ressemblance avec l'approche séquentielle des politiques publiques de Jones est frappante (Jones 1970).
} 
conséquence la démocratie américaine peut encore compter sur le public pour construire l'intérêt général. Mais dans son ouvrage de 1927 Dewey admet que, dans une démocratie de masse où les objets techniques distendent insidieusement le rapport à l'autre et au politique, (trouver le temps de) réfléchir, à plusieurs milliers ou millions et de façon pragmatique, pour élaborer de possibles solutions à des problèmes collectifs n'est pas chose facile. Il propose de faire muer la communauté politique de la démocratie américaine en une «community of inquirers 》: "policies and proposals for social action [should] be treated as working hypotheses, not as programs to be rigidly adhered to and executed [...][Here] the essential need is the improvement of the methods and conditions of debate, discussion and persuasion.» (p.202). On est plus près qu'il n'y parait du concept d'organized intelligence de Lippmann. Pour donner plus de force à sa réhabilitation du public contre Lippmann, John Dewey, dans PIP, ne fait pas des sciences sociales les principales animatrices de la community of inquirers, se contentant de préciser que tous ses membres n'ont pas besoin de savoir enquêter, l'essentiel étant que tout le monde soit capable de comprendre les connaissances produites par d'autres (p.203). Mais il exprime dans un ouvrage de quelques années antérieur et intitulé Democracy and education, an introduction to the philosophy of education (1916) une solide foi dans le positivisme politique des nouvelles sciences sociales : "Every step forward in the social sciences - the studies termed history, economics, politics, sociology -- shows that social questions are capable of being intelligently coped with only in the degree in which we employ the method of collecting data, forming hypotheses, and testing them in action which is characteristic of natural science, and in the degree in which we utilize in behalf of the promotion of social welfare the technical knowledge ascertained by physics and chemistry. Advanced methods of dealing with such perplexing problems as insanity, intemperance, poverty, public sanitation, city planning, conservation of natural resources, the constructive use of governmental agencies for further public good without weakening personal initiative, all illustrate the direct dependence of our important social concerns upon the methods and results of the natural science." (2010 (1916) p.193). Pour le pragmatiste Dewey, il y a lieu de s'inspirer des techniques d'expérimentation scientifique pour donner une armature aux processus politiques de « social problem solving» que sont les politiques publiques.

\section{Charles Merriam, artisan de l'État pragmatique}


Lippmann et Dewey s'accordent donc au moins sur le rôle paragouvernemental des sciences sociales, en dehors du prosélytisme wilsonien en faveur d'un gouvernement bureaucratisé. Ce nouveau rôle pour les SHS américaines après 1918 a son héraut: Charles Merriam. D'ailleurs, dans son livre «New Aspects of Politics» (1925), prélude à sa présidence de l'American Political Science Association, Merriam n'emprunte à Lippmann que l'idée de fonder un «intelligence bureau» dans chaque ministère.

Charles Merriam, fondateur attitré en 1902 du département de science politique de l'université de Chicago, a aussi des ambitions politiques et réformistes locales affirmées (Karl 1974). Il se porte trois fois candidat à la mairie de Chicago - 1912, 16 et 20 - mais échoue à chaque fois, de très peu. La grande différence entre Wilson et lui réside dans le fait qu'il n'a jamais cru que la politique pouvait s'arrêter net aux portes d'une administration publique fût-elle professionnalisée (Merriam 1903). Une science administrative comme enclave au sein de la science politique n'a pour lui aucun sens. Ainsi lorsqu'en 1920, las de ses échecs électoraux répétés, Merriam se retire des courses politiques, il ne renonce pas du tout à la politique, il s'empare simplement d'un nouveau registre d'intervention : les sciences sociales très disciplinées qui lui tendent les bras à l'Université de Chicago. Il écrit ainsi la harangue suivante «... unless a bigher degree of science can be brought into the operations of government civilization is in the very gravest peril from the caprice of ignorance and passion (...). Social science and political science are urgently needed for the next great stage in the advancement of the buman race. » (Merriam 1925 p.295).

Charles Merriam entreprend donc de faire de son département de science politique le nœud de réseau au sein duquel la policy-orientation de sciences sociales américaines sera conçue et connaîtra un début de mise en pratique. Il lance ainsi des recherches dont deux, doctorales, étayent le débat théorique entre Dewey et Lippmann. L'une, avec Harold Gosnell, faisant un large emploi de la statistique, creuse la question de l'abstention (ex : les $50 \%$ d'abstention des élections de Chicago en 1912). L'autre, réalisée par Harold Lasswell, faisant usage de la psychologie, se penche sur la question de la propagande d'État (Lasswell 1927). Dans les deux cas, il s'agit d'esquisser, avec l'appui d'une argumentation scientifique, des politiques de revitalisation de la démocratie américaine, notamment en réponse à la propagande de la toute jeune Union Soviétique qui en remet en question l'authenticité. 
Afin de ressourcer la science politique par d'autres SHS et de flécher leur travail sur des problèmes sociaux réels, Merriam fonde en 1922, avec l'argent des Rockefeller - fondateurs de l'université de Chicago - le Social Sciences Research Council (SSRC). Merriam est identifié à Washington comme la personne charnière entre des sciences sociales de moins en moins spéculatives et une actualité fédérale marquée par la Grande Dépression. F.D. Roosevelt s'appuiera ainsi sur lui pour organiser et animer le National Resources Planning Board dont les études inspireront la fabrique de toutes les politiques publiques du New Deal. L'impact réformateur du NRPB pour le New Deal n'a d'égal que l'animosité qu'il suscite dans la classe politique nationale - ex. des Congressmen acceptant mal d'être cantonnés dans un rôle législatif, la conception et la mise en œuvre des politiques devenant un domaine purement exécutif - comme dans certains segments administratifs - ex. le puissant U.S. Army Corps of Engineers n'appréciant pas l'ingérence des économistes, sociologues et autres politistes dans leurs choix de travaux publics (barrages, routes, digues, réseaux d'électricité...) $)^{7}$. En 1943, la guerre a presque effacé la Grande Dépression et Roosevelt ne peut empêcher la suppression du NRPB voulue par le Congrès. Donc, oui, les sciences sociales «d'Uncle Charlie » - comme Roosevelt aimait à appeler Merriam ont pu être mobilisées pour participer directement et de façon organisée à l'activité gouvernementale fédérale, mais pas de façon durable.

En revanche, Merriam a durablement banalisé l'activité de gouvernement en tant qu'objet de recherche en sciences sociales. Des travaux, qui demeurent de référence, tels "TVA and the Grass Roots» de Philip Selznick (1949) "The American Soldier» de Samuel Stouffer (1949) ou "Administrative Behavior» de Herbert Simon (1947) ${ }^{9}$ participent d'un mouvement de focalisation des sciences sociales sur l'activité gouvernementale réelle (et

\footnotetext{
7 Merriam perd aussi le soutien d'intellectuels, car il a fait trop peu de cas du principe d'autonomie du scientifique par rapport au politique, ou que le NRPB manifeste une confiscation technocratique. Dewey affirme ainsi que le NRPB construit une planned society et non une planning society.

8 Philip Selznick, dans sa thèse sous la direction de Robert Merton à Columbia, montre que la Tennessee Valley Authority, organisation fédérale déconcentrée emblématique du New Deal devant réduire le chômage et la pauvreté dans le Tennessee, se fait aisément et informellement récupérer par les pouvoirs locaux et notamment la caste des grands fermiers et propriétaires terriens, les chômeurs se faisant exploiter et les pauvres restant pauvres.

9 Thèse de doctorat de Simon avec Merriam comme tuteur. C'est en bonne partie grâce à cette thèse que Simon se voit décerner le Nobel d'économie en 1978.
} 
non pas rêvée), mouvement qui s'amplifie après la guerre (Russel Sage Foundation 1950) ${ }^{10}$. L'épigone le plus créatif et hyperactif de Charles Merriam, Harold Lasswell (1903-1978), ne s'y trompe pas qui, dès 1943, alors qu'il dirige l' "Experimental Division for the Study of $W$ ar Times Communications» de la Library of Congress à Washington, donne un nom à cette vague qui enfle : les «policy sciences» (Lasswell 1943).

\section{De l'étatisme pragmatique de Merriam au « policy-scientism » de Lasswell : l'avènement d'une vision instrumentale des politiques publiques}

En octobre 1943, sous la forme d'une lettre qu'il adresse à un petit nombre de collègues, Harold Lasswell fait sa profession de foi en faveur du développement de policy sciences : «2. [...] I desire to contribute to the integration of morals, science and policy. My moral value is that of the individualistic society in which I was reared, and to which I am loyal: the dignity of the human personality. 3. Whatever contribution I make will be scientific and advisory. I am neither equipped to assume an active role nor interested in one for myself. My skill is analysis and creative criticism. 4. My ultimate objective in the field of science is far from modest. I propose to contribute to the systematic theory of the policy sciences. The policy sciences include the social and psychological sciences; in general, all the sciences that provide facts and principles of direct importance for the making of important decisions in government, business and cultural life $^{11} »$ (Lasswell 1943 p.17,).

En 1948, dans Power and Personality, un livre théorique, Lasswell confirme son projet et écrit que les political scientists et les lawyers ne sont pas perçus comme des scientifiques de la démocratie, car leur raison d'être professionnelle, trop procédurale, fait peu de place à une policy orientation instrumentale: c'est à l'épreuve de l'étude des politiques publiques qu'on

\footnotetext{
10 A. Somit et J. Tanenhaus (1967) notent que dans les années 1930, pour de nombreux jeunes docteurs en science politique et en sociologie, la contraction de l'emploi universitaire a été compensée par l'accès à des postes dans les différentes fonctions publiques américaines, renforçant ainsi la légitimité d'une commande publique de recherches adressée au monde académique.

${ }^{11}$ Il me semble qu'on doive interpréter l'évocation du business et de la cultural life comme traduisant une conception extensive, non étatiste, des politiques publiques.
} 
peut véritablement juger du bon fonctionnement d'une démocratie. Comme Merriam, Lasswell s'inspire de Lippmann et de Dewey.

En 1950, dans un article intitulé "Harold Lasswell: Policy Scientist for a Democratic Society ", David Easton, le politiste qui a pris la poste de Merriam à l'université de Chicago et soutient déjà une posture de neutralité axiologique forte en science politique, ironise sur le flou nimbant la conception lasswellienne de la démocratie. Il souligne aussi le décalage entre le démocratisme des policy sciences de Lasswell et son élitisme froid, à la Lippmann, défendu dans son fameux livre Politics: Who Gets What, When, How (Lasswell 1936). ${ }^{12}$

La réponse de Lasswell, vigoureuse, prend la forme d'un ouvrage collectif codirigé avec Daniel Lerner et intitulé : The Policy Sciences: Recent Developments in Scope and Method. Lasswell y précise les contours d'une policy orientation dans les sciences sociales et balaie aussi d'un revers de main le procès en légitimité académique intenté par Easton en convoquant des contributeurs de très haut vol: Kenneth Arrow (économie), Margaret Mead (anthropologie), Robert Merton (sociologie) ou Edward Shils (théorie sociale). Terminée la profession de foi de 1943, place à la formation d'un parti politico-scientifique dont le manifeste se résumerait ainsi :

1) il existe un espace sémantique transdisciplinaire ${ }^{13}$, les policy sciences, dont ressortissent des recherches disciplinaires ou sous-disciplinaires en public administration, government studies, sociology of organizations, economy of industrial relations, psychology of communist leaders..., qui renvoient explicitement à d'importants choix de société par lesquels une collectivité réalise ou non ses grandes aspirations ;

2) les auteurs de ces recherches doivent maintenant prendre conscience qu'ils prennent de facto une responsabilité vis-à-vis du système politique au

\footnotetext{
1255 ans plus tard, trois politistes américains de renom ont fait valoir un droit d'inventaire sur la «policyorientation » de Lasswell préalablement à une revitalisation des policy studies au sein de la science politique américaine (Farr, Hacker \& Kazee 2006). Le titre de leur article, "The Policy Scientist of Democracy: the Discipline of Harold D. Lasswell» est clairement d'inspiration eastonienne.

${ }^{13}$ Lasswell stipule que la science politique ne jouit d'aucun privilège par rapport aux autres disciplines dans le projet des policy sciences, d'où l'usage du terme policy. Evidemment en français ce jeu de langage tombe à plat. Le livre a été rapidement traduit en français sous le titre: le développement des «sciences de la politique » aux États-Unis (1951) (le vocable "politique publique » sera trouvé après). Raymond Aron en signe la préface, très désabusée et dubitative.
} 
sein duquel ils opèrent et qu'en l'espèce les policy scientists américains, pour mériter cette appellation, doivent s'accorder sur de grandes valeurs que la démocratie américaine doit mettre en pratique à travers des politiques publiques et les prendre comme références dans la conduite de leurs recherches ${ }^{14}$; Lasswell cite en exemple l'étude de l'économiste suédois Gunnar Myrdal sur les Afro-Américains (1944) qui, tout en étant scientifiquement irréprochable, explicite clairement pourquoi la situation des Noirs aux États-Unis est proprement scandaleuse pour une démocratie moderne ;

3) la démocratie américaine et ses citoyens ordinaires ont besoin de "practitioners of the sciences of democracy", d'experts bien sélectionnés et identifiés, capables de clarifier les buts poursuivis par les politiques publiques et d'en mesurer précisément la réalisation ; à l'image de nos corps qui ont besoin de médecins pour résoudre nos problèmes de santé (Lasswell 1948).

David Easton (1950), en distinguant dans l'itinéraire intellectuel de Lasswell une «elitist amoral phase » pré-1940 d'une « decisional moral phase» post-1940, exagère la mue idéologique de Lasswell. Celui-ci conserve en effet intact son attachement au modèle élitiste de démocratie tout en considérant que, dans un ordre politique démocratique, le policy scientist peut non seulement faire son travail, mais doit aussi faire partie de ce segment de l'élite au pouvoir qui, grâce à son expertise, est en mesure de guider positivement les choix de politiques publiques des citoyens, à condition que le chercheur en question assume professionnellement ce rôle crucial.

Disons-le d'emblée : les propositions 2) et 3) de Lasswell n'ont guère suscité d'engouement au sein des sciences sociales américaines. Ainsi que le formule en 1975 le politiste Yehezkel Dror, un proche de Lasswell qu'il a rencontré à la Rand Corporation : «La plupart des «policy scientists 》 aux ÉtatsUnis travaillent sur des problèmes concrets avec des techniques spécifiques, négligeant la construction d'une approche intégrée de la Policy Science avec des présupposés philosophiques explicites» (Jaumin-Ponsar 1975 p.28). Les sciences sociales

\footnotetext{
${ }^{14}$ Ce n'est qu'en 1971 qu'Harold Lasswell, dans un livre intitulé «A Pre-View of Policy Sciences» écrira qu'analyser une politique publique c'est interroger l'évolution de la situation sociale problématique traitée et du comportement de ses participants (ressortissants inclus, bien entendu) en référence aux valeurs suivantes : Power, Enlightment, Wealth, Well-Being, Skill, Affection, Respect, Rectitude (Lasswell 1970).
} 
américaines n'auraient pas eu grand intérêt à adhérer ouvertement au pari scientifico-politique de Lasswell. Elles ne rencontraient à l'époque aucun souci de développement, même la National Science Foundation, créée en 1948, les a incluses dans son champ d'intervention. Et puis Lasswell suscite circonspection et méfiance chez beaucoup. Connu pour être le conseiller discret, voire occulte de multiples princes - au département de la défense, à la CIA, à la Rand Corporation, mais aussi au Congrès, aux Fondations Ford et Rockefeller, et même à l'American Civil Liberties Union - son appel à la formation de sciences de la démocratie semble étrange, tant l'acception procédurale ou constitutionnaliste, bref non instrumentale, de la notion de démocratie reste prégnante.

En fait, dans le triptyque que je propose du message de policy-orientation de Lasswell, seul le premier panneau a petit à petit rencontré un écho important dans les SHS américaines. C'est là que l'on peut revenir brièvement sur Merriam. En effet, pour bien comprendre le succès socialscientifique partiel, mais réel de la policy-orientation de Lasswell, il apparait pertinent de comparer ses stratégies de positionnement intellectuel avec celles de son maître Merriam.

\section{Ce que l'élève Lasswell garde et ne garde pas de son maître Merriam}

Merriam ne considère pas la science politique comme une discipline socialscientifique comme les autres. La science politique se ressource auprès des autres sciences sociales sur les plans méthodologique et empirique, mais n'est pas une discipline parasite car elle jouit grosso modo du monopole de valorisation politique de ces ressources scientifiques captées dans les autres disciplines, dans le cadre d'un projet d'Etat organique pragmatique. Il ne s'agit pas de bouleverser mais plutôt d'intégrer nationalement un système politique américain fondé sur une myriade de «small-town politics» constituant autant de creusets de la participation citoyenne essentielle à la conduite des affaires publiques. Pour Merriam, la science politique, à la différence des autres SHS, doit chercher ses applications dans la vie

politique en acceptant d'emprunter un registre non-positiviste de production de connaissances prenant au sérieux les savoirs, les savoir-faire «vernaculaires » des citoyens, de leurs représentants et de leur serviteurs 
bureaucratiques en essayant de les améliorer, plutôt que de s'y substituer (Merriam 1931).

Comme le pointe Douglas Torgerson (1995), Charles Merriam est un aristotélicien convaincu : l'être humain est avant tout un animal politique, il est impossible pour les sciences du politique de dégager des lois universelles et stables régissant tous les comportements politiques des hommes. Les sciences sociales doivent principalement construire leur raison sociale en mettant à disposition des citoyens des connaissances empiriques, produites méthodiquement et concernant des situations d'action publique problématiques. Elles doivent aider les acteurs politiques - du simple citoyen à la plus haute magistrature de la République - à cultiver cette vertu que Merriam nomme la "political prudence » - en traduction du concept aristotélicien de phronèsis - qui recouvre à la fois une sagesse pratique instrumentale - trouver les moyens d'atteindre une fin - et un mélange de pondération et de modestie quant à l'emploi des moyens choisis pour atteindre une fin.

Cernons maintenant comment Lasswell se démarque de Merriam. Déjà, Lasswell ne reprend pas à son compte la démiurgie étatiste servant de colonne vertébrale à l'œuvre de Merriam ${ }^{15}$. La policy orientation de l'élève a supplanté l'étatisme du maître. Pour un intellectuel comme Lasswell, dans un pays qui, dès les années 1930, mais surtout après 1945 et le début de la guerre froide, se livre à un combat à mort avec des États à tendances totalitaires - ex. l'Union soviétique, ne laisser transparaitre aucun dessein étatiste est gage de respectabilité politique et de meilleure écoute de la part de l'élite au pouvoir (Lasswell 1941). Mais l'a-étatisme de la policy orientation de Lasswell trahit aussi un scientisme dans lequel Merriam ne se reconnait pas. Les politiques publiques recouvrent une réalité nettement moins métaphysique que celle de l'État et donc plus facilement appréhendable par des chercheurs sociaux tentant de mimer leurs collègues des sciences naturelles. De surcroît, dans son étatisme, Merriam prend les sciences sociales de haut et séparément les unes des autres, les considérant simplement comme des sources d'inspiration pour l'homme politique

\footnotetext{
${ }^{15}$ Barry Karl évoque dans sa biographie de Merriam les débats publics houleux entre celui et l'économiste autrichien Friedrich Hayek (qui a pris poste à l'université de Chicago en 1950) sur le thème marché versus planification étatique, à la fin des années 1940.
} 
prudent nouveau dont il appelle l'avènement. Cela peut expliquer pourquoi, de l'avis des bons connaisseurs de l'œuvre de Lasswell interviewés par nous en $2001^{16}$, Lasswell considérait que Merriam avait échoué à faire du social science research council un chaudron transdisciplinaire à même de contribuer à la résolution de grandes questions de société. C'est pour abolir toute frontière disciplinaire et ne conférer aucune supériorité relative à la science politique que Lasswell invente le vocable de «policy science » qui diffère donc très sensiblement de la «political prudence » de Merriam.

D'aucuns considèrent aujourd'hui que la surface académique très transdisciplinaire de Lasswell va de pair avec une certaine superficialité scientifique $^{17}$. Mais, de son vivant, après la seconde guerre mondiale et sa titularisation à Yale, cette surface académique s'accompagnait d'une capacité de mobilisation des meilleurs social-scientifiques des États-Unis autour de la culture des policy sciences. La stratégie aristocratique et élitiste, platonicienne, disons-le, de Lasswell est autrement plus valorisante pour les chercheurs en sciences sociales que la petite morgue aristotélicienne à peine voilée que leur démontrait Merriam au SSRC ou au NRPB.

En résumé, paradoxalement, c'est en adoptant une stratégie de positionnement académique et politique radicalement différente de celle de Merriam que Lasswell en perpétue le pragmatisme politique, à la fois volonté et représentation selon lesquelles on doit juger le système politique américain davantage sur son action et son efficace sociétales que sur sa forme. Lasswell recrée le pragmatisme de Merriam en le nommant policy sciences, une appellation scientifiquement et académiquement fédératrice et

\footnotetext{
16 Ronald Brunner (Boulder U., Denver, 12/4/2001), Charles Lindblom, Michael Riesman et Andrew Willard (Yale U., New Haven, 16/4/2001).

17 Autant Merriam et le nouvel homme politique, prudent, inspiré par les sciences sociales, qu'il veut voir éclore, se ressemblent, autant Lasswell incarne avec éclat le policy scientist, ce génie transdisciplinaire apte à examiner les politiques publiques sous toutes coutures. Enfant surdoué chéri par des parents excellents pédagogues, Lasswell entre en 1918 à l'Université de Chicago à 16 ans, d'abord en sociologie, puis en économie (Bachelor), puis en science politique $(\mathrm{PhD})$ sous la direction de Merriam. Celui-ci le fait travailler sur les questions de propagande en croisant psychologie, psychosociologie et la quantification des actes médiatiques (invention de "l'analyse de contenu ») et l'envoie grâce aux fonds du SSRC glaner des idées en Europe, à Londres, Paris, Genève et Berlin où il entre en analyse avec un disciple de Freud. De cet investissement dans la psychanalyse sortira un livre qui fera scandale, « Psychopathology and politics» (1930), car traversé par une question d'eugénisme politique: «comment faire pour avoir enfin moins de psychopathes au pouvoir ? (Muth et al. 1989).
} 
mobilisatrice $^{18}$, et à l'apparence politiquement inoffensive : les policy scientists, comme leur nom l'indique, font de la science, pas de la politique.

\section{La consécration en demi-teinte de la policy-orientation}

Avec la publication de l'ouvrage The Policy Sciences : Recent Development in Scope and Method (1951), l'espace sémantique délimité par Lasswell voit arriver des lotisseurs politiques ou académiques, pas toujours en phase d'ailleurs avec la vision platonicienne de Lasswell, tel Charles Lindblom qui, dans son article de 1958 "The science of muddling through» affirme que la policy science habite probablement moins les scientifiques que les policy-makers, petits et grands, interagissant prudemment et de façon gradualiste et décousue (disjoint incrementalism). L'intelligence de Lasswell consiste aussi à illustrer son idée de policy orientation de façon très opportune. Ainsi, en 1950, en plein guerre froide et début du maccarthysme, poursuivant ses sombres réflexions sur l'avènement de " garrison states ${ }^{19}$ » (Lasswell 1941), Lasswell publie " National Security and Individual Freedom » où il alerte «l'opinion et ses leaders » sur les dangers encourus par la démocratie américaine si la sécurité nationale en devenait l'obsession ${ }^{20}$. Grâce à ce type d'illustrations, basées sur une actualité brûlante, une phrase comme "The policy sciences approach calls forth a very considerable clarification of the value goals involved in policy " (Lasswell 1951, p15.) est prise très au sérieux. En janvier 1961, juste avant de céder sa place à John F. Kennedy, le Président Eisenhower adresse ses adieux à la Nation l'enjoignant, à la stupéfaction générale, à la vigilance quant à l'influence grandissante de l'establishment militaro-industriel sur les politiques publiques fédérales. Le principal rédacteur de discours d'Eisenhower est Malcolm Moos, un politiste très friand des écrits de Lasswell. Un an plus tôt, Eisenhower a mis sur pied une "Commission on National Goals", bipartisane, dont le rapport, « Goals for Americans » inspirera à JFK une partie

\footnotetext{
${ }^{18}$ L'éclosion dans les années 1950 et 1960 d'appellations concurrentes, policy analysis, policy research, policy studies,... signe autant des divergences avec le projet de Lasswell qu'un accord sur l'importance de mettre les politiques publiques au centre de l'agenda des sciences sociales américaines..

${ }^{19}$ États se professionnalisant et se spécialisant dans l'exercice de la violence.

${ }^{20} \mathrm{Du}$ coup, aujourd'hui en Amérique, Lasswell est de nouveau convoqué par ceux qui disent que la lutte anti-terroriste est aussi dangereuse pour la démocratie que le terrorisme.
} 
de son programme. Bref, la policy orientation imprègne l'actualité académique et politique.

La consécration institutionnelle de cette dernière advient dans les années 1960 à la faveur du fort interventionnisme des administrations Kennedy et Johnson. Deux épopées scientifiques catalysent cette consécration. La première, appelons-la Bauer, entamée en 1962, porte sur l'impact social et culturel des programmes spatiaux de la NASA. La seconde, appelons-la Coleman, commencée en 1964, a trait à l'efficacité du système scolaire américain.

L'étude Coleman ${ }^{21}$, du nom du sociologue quantitativiste James Coleman (élève de Lazarsfeld) qui l'a supervisée, est commanditée par le Department of Health, Education and Welfare (DHEW) alors en prise avec la délicate application du volet anti-ségrégationniste du Civil Rights Act de 1964 en milieu scolaire. Que démontre cette étude basée sur la passation de tests à près de cent milles élèves et collégiens américains ? : 1) Le milieu socioéconomique de l'élève pèse beaucoup plus dans ses performances scolaires que les moyens proprement éducatifs (heures d'enseignement, infrastructures scolaires, niveau des enseignants, etc.) alloués ; 2) les classes mixtes voient les résultats scolaires des Noirs tirés vers le haut sans détérioration des résultats des Blancs. Les conséquences du rapport Coleman sur la gestion du système éducatif sont telles (affirmative action, desegregation buses, racial quotas, ...) qu'elles valident la thèse d'Edward Shils (1955) selon laquelle la désidéologisation progressive des sciences sociales n'a en rien diminué leur potentiel de contribution à la vie de la Cité. Bref, envisager la formation de policy sciences n'a plus rien d'utopique (Coleman 1982)).

L'étude de Raymond Bauer, psychosociologue soviétologue anticommuniste d'Harvard, commanditée en 1962 par la NASA (créée en 1958 après le traumatisme Spoutnik) porte sur l'impact social et culturel des coûteux programmes spatiaux de cette agence. En échafaudant son enquête Bauer s'aperçoit de son infaisabilité car, après 1945, aucun système national de «comptabilité sociale» n'a été développé à l'instar du système de comptabilité économique mis en place sous le New Deal par le National

${ }^{21}$ Equality of Educational Opportunity Study, 1966. 
Burean of Economic Research, et renforcé en 1946 sous la houlette du Council of economic advisers du Président. En 1964, Bauer apprend que le président Johnson a validé la proposition de son ministre de la Défense, Robert Mac Namara, d'aider ses collègues, dont celui du DHEW, à se doter du Program Planning Budgeting System (PPBS), conçu en 1962 par la Rand Corporation pour rationaliser l'effort de guerre américain au Viêt-Nam. Le sang de Bauer ne fait qu'un tour. Dans le cadre de sa "war on poverty", Johnson lance tout azimut de vastes programmes sociaux, mais ne trouve rien de mieux que des bureaucrates budgétaires pour concevoir, piloter et évaluer ces programmes $^{22}$. Il décide d'exprimer sa frustration sur la place publique en sortant un livre intitulé «Social Indicators» (1966). Au même moment, Bertram Gross, le politiste fondateur du President's Council of Economic Advisors en 1946, publie "The state of the nation, social systems accounting》. S'engage dès lors une intense controverse politique, administrative et scientifique sur la question de savoir de quelles données sociales la sphère fédérale a besoin pour évaluer ex ante et ex post les politiques fédérales (Spenlehauer 1998). Cette controverse a pour effet durable de fixer le vocable "public policy » comme désignant un fragment d'action gouvernementale appréhendable, analysable et évaluable par les sciences sociales (Lowi 1964).

Mais c'est une controverse plus confinée, celle de la remise en cause de l'utilité gouvernementale du PPBS, qui déclenche véritablement le processus d'institutionnalisation de la policy orientation de Lasswell. Le principal artificier est Aaron Wildavsky. Grand spécialiste de la procédure budgétaire (Wildavsky 1964), déjà fort connu pour penser et dire que le PPBS et son systématisme procédurier empêchent de bien réfléchir sur ce que les ÉtatsUnis font au Viêt-Nam, Wildavsky est appelé en 1969 à témoigner devant le Committee on Governement Operations du Sénat américain. Contre toute attente, plutôt que de descendre en flamme l'ensemble du PPBS, il conseille de se débarrasser de sa superstructure, la procédure, et de conserver son infrastructure, les personnels dédiés, pour enfin doter les différents ministères de capacités de policy analysis. ${ }^{23} \mathrm{Il}$ est dès lors moins question de

${ }^{23}$ Le texte de l'allocution de Wildavsky, intitutée "Rescuing policy analysis from PPBS », ne fait pas usage de l'appellation «policy sciences», mais fait bien référence à Lasswell en tant que fondateur de la "policy- 
favoriser une orientation "politiques publiques » dans les sciences sociales académiques que de réussir à former des policy analysts capable of «speaking truth to power», titre du fameux ouvrage publié par Wildavsky en $1978^{24}$. L'idée fait vite son chemin, par exemple à Harvard où la famille Kennedy voudrait bien financer une modernisation de son quelque peu assoupi Institute of Public Administration (entretien avec J.Q. Wilson, 20/2/2001, Los Angeles)(Allison 2006) ou à Berkeley où Wildavsky n'en peut plus des querelles au sein du département de science politique qu'il dirige (entretien avec M. Laudau, 9/4/2001, Oakland) .

En 1971, Harold Lasswell, devenu « Ford Foundation Professor Emeritus of Law and Social Sciences» à la Yale Law School, publie une somme d'allure testamentaire et prospective «A Pre-View of Policy Sciences». Le premier mot $\mathrm{du}$ titre étonne encore. Le message est pourtant clair. La policy orientation a fait beaucoup d'émules en économie, en sociologie, en science politique, en histoire, mais pas de façon transdisciplinaire. Pour Lasswell, en 1971, la formation des policy sciences laisse encore beaucoup à désirer. Le dernier chapitre de cet ouvrage, "Professional Training» présente une maquette détaillée de ce que pourrait être, dans une université généraliste donnée, un dispositif de recherche et d'enseignement en matière de politiques publiques, dispositif articulé autour d'un policy sciences center ${ }^{25}$ et d'une school of policy sciences.

Ô, hasard! en 1972, la Fondation Ford annonce qu'elle finance la mise sur pied de graduate $^{26}$ schools of public policy, pour instruire de "young people aimed at public service », dans sept grandes universités américaines (Berkeley, CarnegieMellon, Duke, Harvard, Michigan, Stanford, Texas), plus une au sein du Rand Institute à Santa Monica. Au cours de la dizaine d'années qui suivent la décision séminale de la fondation Ford, on assiste à une floraison d'écoles ou de master programs of public policy dans près de cent cinquante universités. A

orientation». Wildavsky a fait sa thèse de science politique à Yale à la fin des années 1950 et a été beaucoup influencé par Charles Lindblom.

${ }^{24}$ Le titre complet «Speaking truth to power, the art and craft of policy analysis » pointe ce juste milieu entre une posture élitiste et platonicienne à la Lasswell et une posture lindblomienne où la policy analysis ne revendique pas de statut académique.

${ }^{25}$ Le «center for advanced study of the behavioral sciences » de Stanford est le modèle d'inspiration.

${ }^{26}$ Dotées d'une faculté propre et délivrant diplômes de master et de $\mathrm{PhD}$. 
un rythme de quelques centaines par an, les étudiants frais émoulus des schools or programs of public policy ont rapidement investi les administrations publiques à tous les niveaux de gouvernement américains, branches législatives incluses (on pense notamment au Government Accountability Office du US Congress), les bureaux de lobbyistes, les NGO délégataires de service public (dans le domaine du logement social, par exemple), les think tanks et, last but not least, les cabinets d'évaluation de politiques publiques qui, dès la chute officieuse du PPBS en 1969 (Schick 1973), ont commencé à pulluler (Nachmias 1980 ; Wildavsky 1985).

\section{Puisqu'il faut conclure}

Plusieurs générations de politistes américains, de Wilson à Wildavsky, se sont investies, chacune à sa manière, dans la formation d'un Etat pragmatique résolvant des problèmes sociaux, et ce, en prenant toutes garde à ce que la chose publique construite à l'occasion ne ressemble pas au Léviathan de Hobbes, cette chose terrifiante et surplombante qui hante l'imaginaire politique états-unien. Le retour sur investissement à partir de 1970 est une situation caractérisée par un grand dispositif de production de connaissances évaluatives. Deux traits de ce dispositif impressionnent positivement. Primo, une pluralité d'approches et une inventivité méthodologique sans pareil : du quasi-experimental design à la theory-driven evaluation et au concept d'evaluation policy (Trochim 2009) en passant par la pluralistic \& utilization-focused evaluation, la plupart des outils composant la boîte à outils mondiale de l'évaluation de politique sont "made in USA». Secundo, une vivacité et une multipolarité industrielles: trois grands archipels interagissent pour produire de la connaissance évaluative toutsecteur : l'archipel gouvernemental (exécutif et législatif (ex : GAO)) multiniveaux, l'archipel universitaire ou académique, et entre les deux déjà cités l'archipel non moins resserré des think tanks (Brookings institution (centre), American Enterprise Institute (droite dure), Institute for Policy Studies (gauche radicale)...).

Autrement dit, en termes de capacités analytiques et évaluatives (Howlett 2011), le système politico-administratif américain arbore une belle majesté, et l'on aurait tort de ne pas s'en inspirer de ce côté-ci de l'Atlantique, où l'on observe encore trop souvent des acteurs qui convertissent, sans 
apprentissage ni modestie, leur légitimité politique et/ou institutionnelle en légitimité évaluative (ex: le Comité de contrôle et d'évaluation des politiques publiques de l'Assemblée nationale), avec une plus-value d'amélioration de l'action publique proche de zéro. Serait-ce si compliqué de disposer de plus de gens et de services sachant construire un evaluanda, composer une instance d'évaluation dynamique, constituer et interroger des échantillons de ressortissants pour mesurer des effets d'aubaine, imaginer des schémas organisationnels alternatifs pour la politique évaluée...?

Serait-ce alors à dire qu'il faille une nouvelle fois se lamenter sur le retard français par rapport à nos cousins d'Amérique ? Pas si sûr. Les approches évaluatives économiques, et plus largement celle ne se souciant pas de l'éventuelle et souhaitable utilisation gouvernementale des connaissances produites demeurent dominantes (Mead 1983; Vedung 2010). Et ce fréquent refoulement du politique par les praticiens américains de l'évaluation indique que, contrairement aux dires de Nelson Polsby (1983), la policy evaluation américaine a bien davantage épousé les formes du jeu politique de ce pays qu'elle ne les a éclairées et transformées, avec, en d'autres termes, une politics of knowledge (Lagemann 1987; Lindblom 1990 ; Smith 1991) directement dérivée d'une politics of interest groups (Berry \& Wilcox 2007). Le French way of policy evaluation garde donc toutes ses chances!

Allison G., "Emergence of Schools of Public Policy: Reflections by a Founding Dean", in M. Moran, M. Rein, R.E. Goodin, eds. 2006, The Oxford Handbook of Public Policy, Oxford University Press.

Bauer R.A. (Ed.) 1966, Social Indicators, MIT Press, Cambridge (Mass.).

Berry J.M., Wilcox C. 2007, The Interest Group Society, Pearson Longman, New York.

Cassirer E. 1944, The Myth of the State, Yale University Press, New Haven (CT) (Trad. Française, Gallimard 1993).

Coleman J.S., , "The Emergence of Sociology as a Policy Science”, in Coser L.A., Larsen O.N. 1982, The Uses of Controversy in Sociology, The Free Press, New York, pp.253-261.

Dewey J: 
- 1927, The Public and Its Problems, The Swallow Press, Chicago (Ill.) (Reprint, 1954);

- 1916, Democracy and education, an introduction to the philosophy of education, The Macmillan Company, NY (NY);

- 1910, How We Think, D.C. Heath \& Co. Publishers, New York (NY).

Easton D. 1950, "Harold Lasswell, Policy Scientist for a Democratic Society", The Journal of Politics, Vol. 12, No. 3 (Aug., 1950), pp.450-477.

Farr J., "Political Science and the State", in Farr J., Seidelman R. (eds.) 1993, Discipline and History. Political Science in the United States, the University of Michigan Press, Ann Arbor, pp. 63-80.

Farr J., Hacker J., Kazee N. 2006, "The Policy Scientist of Democracy: The Discipline of Harold D. Lasswell", American Political Science Review, 100:4 579-587

Gunnell J.G., "Continuity and innovation in the history of political science: the case of Charles Merriam”, J. of the History of Behavioral Sciences, vol. 28 April 1992 pp. 133-42.

Gross B. 1966, The State of the Nation: Social Systems Accounting, Tavistock Publications, New York (NY).

Hamelin F., Spenlehauer V., «Road Policing as a state tool. What tells the genesis of the California Highway Patrol » (25 p.), Policing and Society, vol. 16, n³, September 2006, pp. 261-284.

Haskell T.L. 2000, The Emergence of Professional Social Science, John Hopkins University Press, Baltimore (Mar.).

Hofstadter R. 1955, The Age of Reform. From Bryan to F.D.R., Vintage Books, NY (NY).

Howlett M. 2011, Designing Public Policy: Principles and Instruments, Routledge, New York

Jaumin-Ponsar A 1975, La policy science: Une nouvelle école de la décision publique, Bruylant, Bruxelles.

Jones Ch. O. 1970, An Introduction to the Study of Public Policy, Duxbury Press, Belmont.

Karl B.D. 1974, Charles E. Merriam and the study of politics, University of Chicago Press, Chicago.

Lagemann E.C. 1987, The Politics of Knowledge. The Carnegie Corporation, Philanthropy, and Public Policy, University of Chicago Press, Chicago (Ill.).

Lasswell H.D.:

- 1927, Propaganda Technique in the World War, Knopf, NY (NY);

- 1930, Psychopathology and Politics, University of Chicago Press, Chicago (Ill.);

- 1936, Politics: Who gets What, When, How, McGraw-Hill, NY (NY);

- 1941 «The Garrison State », American Journal of Sociology, January, 46, pp. 455-68;

- "Memorandum: Personal Policy Objectives", October 1943, Harold Lasswell papers, Yale University Library, New Haven (CT);

- 1948, Power and Personality, Norton \& Co., New York 
- with Lerner D. (eds.) 1951, The policy sciences: recent developments in scopes and methods, Stanford University Press, Palo Alto (Ca);

- 1971, A Pre-View of Policy Sciences, Elsevier, NY (NY).

Lindblom Ch.:

- «Policy Analysis », American Economic Review, 48 (June), 3:1958 pp.298-312;

- "The Science of "Muddling-Through"”, Public Administration Review, 195919 (Spring) pp.79-83;

- 1990, Inquiry and change. The troubled attempt to understand and shape society, Yale University Press, New Haven (CT).

Lippmann W. 1922, Public Opinion, Harcourt, Brace, NY (NY).

Lowi Th.,

- "American Business, Public Policy, Case-Studies, and Political Theory", World Politics, 1964 16(4), pp.677-715

- "The state in political science: how we become what we study", American Political Science Review, 1992 vol.86 pp1-7.

Masuoka, N., Grofman B, Feld S. 2007, "The Production and Placement of Political Science Ph.D.s, 1902-2000” PS: Political Science and Politics. 40(2): 361-366.

Merriam Ch. E.:

1903, A History of American Political Theory, MacMillan, NY (NY);

1925, 1931, New Aspects of Politics, University of Chicago Press, Chicago (Ill.)

Mills Ch. W. 1956, The Power Elite, Oxford University Press NY (NY).

Muth R., Finley M., Muth M. 1989, Harold D. Lasswell: an Annoted Bibliography, New Haven Press, New Haven (CT).

Myrdal G. 1944, An American Dilemma: The Negro Problem and Modern Democracy, Harpers \& Brothers, NY (NY).

Nachmias D.: "The Role of Evaluation in Public Policy", Policy Studies Journal, 8:1980, pp.1163-69

Novak W.J., "The Myth of the «Weak» American State», American Historical Review, 113:2008 pp.752-72.

Payre R., Pollet G. 2013, Sociohistoire de l'action publique, La Découverte (coll. Repères), Paris.

Polsby N. 1983, “Tank but no tanks”, Public Opinion, April/May pp.14-58.

Ricci D. 1984, The Tragedy of Political Science: Politics, Scolarship, and Democracy, Yale University Press, New Haven (CT).

Russel Sage Foundation 1950, Effective Use of Social Science Research in The Federal Services, Russell Sage, New York (NY). 
Schick A., "A death in the bureaucracy: the demise of federal PPB", Public Administration Review, 33 (2), March/April 1973, pp. 146-56.

Selznick Ph. 1949, TVA and the Grass Roots, a Study in the Sociology of Formal Organization, University of California Press, Berkeley and Los Angeles (Ca).

Simon H. 1947, Administrative Behavior, the Free Press, New York.

Shils E., “The end of ideology?” Encounter, 5 (November 1955) pp.52-58.

Skowronek S. 1982, Building a New American State; the Expansion of National Administrative Capacities 1977-1920, Cambridge University Press, Cambridge (UK).

Smith J.A. 1991, The Idea Brokers: Think Tanks and the Rise of the New Policy Elite, The Free Press, New York (NY).

Somit A., Tanenhaus J. 1967, The Development of American Political Science, from Burgess to Behaviourism, Allyn \& Bacon, Boston (Mass.).

Spenlehauer V. 1998, L'évaluation des politiques publiques, avatar de la planification, Thèse de doctorat de Science Politique, UPMF Grenoble II (consultable sur http://hal.archivesouvertes.fr $/$ )

Stouffer S.A. 1949, The American Soldier, Studies in Social Psychology in World War II, Princeton University Press, Princeton (NJ).

Trochim, W.M.K. (2009), "Evaluation policy and evaluation practice”, in W.M.K.

Trochim, M. M. Mark, \& L. J. Cooksy (Eds.), Evaluation policy and evaluation practice. New Directions for Evaluation, 123, 13-32.

Torgerson D., "Policy Analysis and Public Life: the Restoration of Phronèsis", in Farr J., Dryzek J.S., Leonard S.T. (eds.) 1995, Political Science in History: Research Programs and Political Traditions, Cambridge University Press, Cambridge (UK), pp. 225-252

Wildavsky A.:

- 1964, Politics of the Budgetary Process, Little Brown \& Co, Toronto (Can).

- "Rescuing Policy Analysis from PPBS", Public Administration Review 29 (2) 1969 pp.189-202

- 1979, Speaking Truth to Power. The Art and Craft of Policy Analysis, Little Brown \& Co, Boston (Mass.)

- " "The once and future school of public policy", The Public Interest, $1985 \mathrm{n}^{\circ} 79$ pp. $25-41$

Wilson W., “The study of administration”, Political Science Quaterly, vol.2 June 1887, pp.197-222. 